\title{
Sitzung vom 25. Januar 1897.
}

Vorsitzender: Hr. F. Fischer, Vice-Präsident.

Das Protocoll der letzten Sitzung wird genehmigt.

Zu ausserordentlichen Mitgliedern werden verkündet die Herren:

Bakhuis-Roozeboom, Prof. H., Amsterdam;

Stoklasa, Dr. J., Prag;

Johnson, Prof. Dr. S. W., New Haven;

van der Meulen, Dr. P. H.,

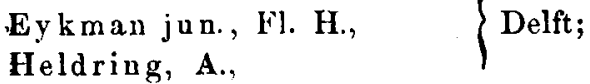

Kã̃er, K., Genf:

Kjellin, Dr. C., Mõlnlycke;

Fischer, A.,

Feith, H., $\}$ Heidelberg;

Merz, A.,

Micko, Dr. K., Wien;

Harz, Dr. K., Kulmbach;

$\left.\begin{array}{l}\text { Kölle, M., } \\ \text { Völter, P., }\end{array}\right\}$ Tübingen;

Hefelmann, Dr. R., Dresden;

Schimmel, H., Charlottenburg;

Davis, W. A., London;

Findlay, A., Aberdeen;

Mayer, F., Mainz;

w. Bitti, Budapest.

Zu ausserordentlichen Mitgliedern werden vorgeschlagen die Herren:

Hoyer, Emil, Schillerstr. 118, IV, Charlottenburg i(durch C. Liebermann und H. Finkenbeiner); 
Tepelmann, Bernh.., is Fa.: Friedr. Vieweg Sohn, Braunschweig (durch H. Landolt und K. v. Buchka)!

Holtmannn, Dr. A., Grunthensthalstr. 16, Freiburg i. Br.

(durch C. Willgerodt und A. E.dinger);

Conrad, Erich, Plattenstr. 19, Zürich $\mathbf{V}$ (dureh $A$. Werner und C. Scball);

Kippenberger, Prof. Dr. C., Kairo (durch L. Knorr und L. Wolff):

Fritz, Dr. Sigmund, Akademiestr. 1, Heidelberg (durch V. Meyer und L. Gattermann);

Bruck, Dr. Carl, Magister der Pharmacie, Liechtensteinstrasse 32/34, Wien IX (dureh E. Lippmann und P. (ohn);

Wright, A. C., 44 Margaretstr., Hull (durch A. Smitbells und B. Cohen);

Meiser, W., Liebigstr. 18, Leipzig (dureh B. Rassow und P. F. Schmidt);

Klages, Ludwig, Augustenstr. 30 III, München (durch A. Einborn und W. Königs);

Harris, C. B., 4804 St. Lawrence Avenue, Chicago (durch A. Noyes und H. Bullard);

Schwerin, Graf von, Schiffbauerdamm 35,

Stock, Alfred, Artilleriestr. 29,

Hoffa, E., Pestalozzistr. 5, Charlottenburg, O. Ruff ); Froebe, Dr. Wilhelm, Wasagasse 20, Wien IX (durch

A. Lieben und R. Wegscheider);

Mitscherlich, Sigurd, Chem. Institut, Rostock (durcb

A. Mitscherlich und E. Fritzweiler);

Grübler, Dr. Georg, Bayerschestr. 63, Leipzig (durch

M. Siegfried und P. Balke);

Kramer, (i., IOôtel Terminus, Freiburg (Schweiz) (durch

A. Bistrzycki und H. Finkeubeiner);

Borsche, Walter, Walkmühlenweg 9, Göttingen (durch

O. Wallach und W. Kerp);

Nass, Dr. G., Güthestr. 71 I, Charlottenlourg (durch A. Sitavenhagen und $O$. Kühling);

Goppelt, Iudwig, Louisenstr. 10,

Prall. Friedrich, Strandstr. 98, Freundlich, Sally, Neue Werderstr. $4 a^{\prime} I$, A. Michaelis Stoppelhaar, G., Friedhofsweg. und

Ortmeyer, Paul, Neue Werderstr. 39, R.Stoermer); $\mathrm{Nahter}$, A lexander, Wismarschestr. 30 II, 
v. Raumer, Dr. Eduard, Schlosegraben 1,

Späth, Dr. Eduard, Oestl. Stadtmauerstr. 20 ,

Erlangen (durch

E. Beckmann und

H. Reckleben);

Ragland, C. D.,

Chamberlat in, J. S., Johns Hopkins University, Baltimore, Magruder, E. W.,

Waters, C. E., Md. (durch Ira Remsen und Edw.

Der Schriftführer verliest den unten abgedruckten Anszug aus dem Protocoll der Vorstands-Sitzung rom 19. Januar 1897.

Der Bibliothekar theilt mit, dass das neue Lesezimme cer Bibliothek (vgl. diese Berichte 29, 2859) am Montag, den 1. Februar d. J. der Benutzung übergeben werden wird.

Für die Bibliothek sind als Geschenke eingegangen:

796. Cohn, Georg. Tabellarische Uebersicht der Pyrazolderivate. Brannschweig 18.97 .

797. Cannizzaro, S. Scritti intorno alla teoria molecolare ed atomica ed alla notazione chimica. Palermo 1896.

Der Vorsitzende:

E. Fischer.
Der Schriftühbrer :

A. Pinner.

\section{Auszug aus dem \\ Protocoll der Vorstands-Sitzung \\ vom 19. Januar 1897.}

Anwesend die Herren Vorstandsmitglieder: E. Fischer, S. G a briel, J. H. van't Hoff, J. F. Holtz, E. Jacobsen, G. Kraemer, B.Lepsius, C. Liebermann, A. Pinner, C. Schotten, H. Thierfelder, F. Tiemann, H. Wichelhaus, W. Will, sowie der Generalsecretär Hr. P. Jacobson.

4. Zu Mitgliedern der Publications-Commission werden die Herren H. Landolt, C. Liebermann, A. Pinner, F. Tiemann und $\mathrm{H}$. Wichelhaus wiedergewählt.

5. Zum Redacteur wird Herr F. Tiemann wiedergewählt. 